\title{
Post-tetanic mechanical tension and evoked action potentials in McArdle's disease
}

\author{
N.J. B R A N D T, F. B U C H T H A L, F. E B B ES E , Z. K A M I E I E C K A, \\ A N D C. K R A R U P
}

From the Laboratory of Clinical Neurophysiology, the Department of Pediatrics, Section of Teratology of the Rigshospital, and the Institute of Neurophysiology, University of Copenhagen, Denmark

SUMMARY The tension produced by the cramp evoked in the adductor pollicis muscle by repetitive stimuli to the nerve $(20 / \mathrm{s}$ for $50 \mathrm{~s})$ and by full voluntary effort in the brachial biceps was measured in a patient with McArdle's disease. The contracture was $17 \%$ of the peaktetanic tension, and was not associated with action potentials. Twitches superimposed on the contracture were at most diminished to half, as were their action potentials. Both slow and fast muscle fibres participated in the contracture. The contraction time of the twitches elicited after the tetanus was prolonged more in the patient than in a normal subject of the same age. There was evidence of delayed firing, first observed 90 seconds after the peak of the contracture. The patient had electromyographic and histological signs of myopathy.

McArdle's disease is associated with painful muscle cramps and myoglobinuria after strenuous exercise (McArdle, 1951; Rowland et al., 1963). The cramps are not accompanied by electrical activity (McArdle, 1951). The strength of the contracture has not previously been measured. In the patient presented here we have determined the force developed during the contracture. As a measure of the number of fibres that do not contribute to the contracture, we recorded the muscle action potential and the twitch evoked during the contracture by stimulating the nerve.

\section{Method}

\section{STIMULATION}

The ulnar nerve was stimulated supramaximally at the wrist after local anaesthesia at the elbow (4 $\mathrm{ml}, 1 \%$ lidocain). Insulated steel needles with a $3 \mathrm{~mm}$ bared tip were placed along the nerve. The threshold of the action potential of $\mathrm{m}$. adductor pollicis was used as a gauge of optimal placement. The threshold was $0.3 \mathrm{~mA}(0.2 \mathrm{~ms}$ pulses $)$, and the electric current used to stimulate maximally was $28 \mathrm{~mA}$ to ascertain activation of all motor fibres

Address for reprint requests: Professor F. Buchthal, Laboratory of Clinical Neurophysiology, Juliane Maries Vej 36, DK 2100 Copenhagen, Denmark.

Accepted 29 April 1977 even if there should be some displacement of the electrodes during the tetanic contraction.

The contracture was evoked by stimuli given at a rate of 20/s for $50 \mathrm{~s}$ (Dyken et al., 1967). In addition, single responses were evoked before, during, and after the contracture and, to produce a staircase phenomenon, $2 / \mathrm{s}$ trains of stimuli were applied for $90 \mathrm{~s}$.

\section{RECORDING}

Adductor pollicis muscle The muscle action potential was recorded via a steel needle of low impedance placed under the skin above the belly of the muscle. The remote electrode was placed on the surface of the distal phalanx of the thumb. The action potentials were amplified (DISA 9014C0101) and recorded via an event recorder on an ink jet writer (Mingograph, Siemens), the resulting frequency range being $20-10000 \mathrm{~Hz}$ (Dahl and Buchthal, in preparation).

The mechanical force was recorded isometrically on one channel of the ink jet writer (frequency $0-1000 \mathrm{~Hz}$ ) by a mechano-electrical transducer (Slomić et al., 1968). To prevent artefacts, the hand and the forearm were rigidly fixed. In control experiments it was ascertained that the drift in resting tension was less than $30 \mathrm{~g}$ for the duration of the examination (15 minutes) - that is $1 \%$ of the maximum tetanic tension and $6 \%$ of the maximum tension during the contracture. 
Flexors of the elbow (examined during maximum voluntary effort) Action potentials were recorded via concentric needle electrodes (DISA 13L31) inserted into the brachial biceps muscle. The isometric force at the wrist was recorded with the elbow joint at an angle of $60^{\circ}$.

The surface temperature of the upper limb was kept at $38^{\circ} \mathrm{C}$ by an automatically controlled infrared heating element.

\section{PROCEDURE}

After the tetanus was recorded, the contracture and the twitches and their action potentials were recorded at 1-2 minute intervals using a sweep speed of $200 \mathrm{~ms}$ per $\mathrm{cm}$. In addition, the electrical responses and the mechanical force were recorded continuously at a speed of $500 \mathrm{~ms}$ per $\mathrm{cm}$.

\section{ELECTROMYOGRAPHY}

The pattern of the electrical activity was recorded via concentric electrodes during full effort. The mean duration of motor unit potentials, their mean amplitude, and the incidence of polyphasic potentials were determined from 40-60 different motor unit potentials during weak effort in the brachial biceps and the medial vastus muscles.

\section{Description of the patient}

The boy was 13 years old; his parents were unrelated; he had an older brother who died at the age of 6 years without signs and symptoms of neuromuscular disease. There was no history of neuromuscular disease in the family. The motor impairment of the patient was noticed at 2 years of age. In the past five years he had eight episodes of cramps, muscle pain, and myoglobinuria after heavy exercise. The recent attacks involved any muscles, including masticatory muscles, and were evoked by moderate exercise. At present he can walk without cramps but not run. Pain and stiffness of muscles provoked by moderate exercise may disappear when the exercise is continued ('second wind' phenomenon, Porte et al., 1966).

Neurological examination showed neither weakness nor wasting in the upper or lower extremities or in the facial muscles. Neck flexion was performed with markedly diminished force. Sensitivity to touch, pin prick and vibration, postural sensation and tendon jerks were normal.

Serum enzymes were determined two and five months after an episode of muscle cramps associated with myoglobinuria. Creatine kinase was four and ten times above the upper limit of normal; lactate dehydrogenase was once two times normal and another determination was within normal limits; the increase of its isoenzyme 5 was more marked, ten times normal.

Muscle enzymes (determined by Dr P. Wang): phosphorylase activity was virtually absent. There was definite activity of phosphorylase kinase and of glycogen synthetase (Krebs et al., 1964; Thomas et al., 1968).

Ischaemic exercise (Ebbesen et al., 1977) was associated with cramps and pain in forearm muscles. The concentration of lactate and pyruvate in venous blood was the same after as before the exercise (Landon et al., 1962; Lundholm et al., 1963).

Biopsy of the brachial biceps muscle showed slight changes that indicated myopathy: $4 \%$ of the fibres had internal nuclei; $1-2 \%$ of the fibres were necrotic or showed signs of regeneration (large nuclei, basophilia). There was slight proliferation of connective tissue between muscle fibres. The average diameter of muscle fibres was slightly diminished and the variation of fibre diameters was normal (Fig. 1).

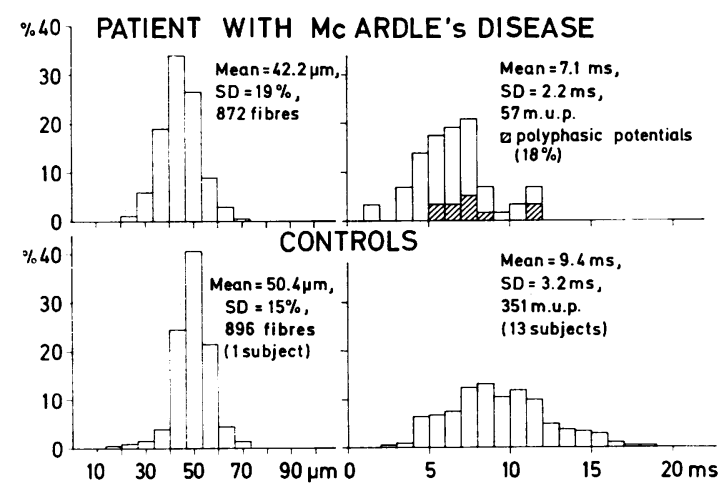

Fig. 1 Distribution of muscle fibre diameters (left) and of the duration of different motor unit potentials (m.u.p., right) in the brachial biceps muscle of patient with McArdle's disease (above) and of controls matched for age (below). The mean diameter was diminished and the mean duration was shortened $(\mathrm{P}<0.001)$.

\section{HISTOCHEMISTRY}

The specimen was examined for active phosphorylase $a$ and inactive phosphorylase $b$ in the presence of adenosine monophosphate (AMP). In addition, inactive phosphorylase $b$ was activated by phosphorylase kinase in the presence of adenosine triphosphate (ATP) and Mg-ions (Eränko and Palkama, 1961; Godlewski, 1963). For control, a section of a normal muscle was examined on the same cover glass. Phosphorylase $a$ and $b$ were absent; only the smooth muscle cells of the intra- 
muscular blood vessels had traces of phosphorylase activity. The muscle fibres stained heavily for glycogen and many fibres showed in addition conglomerates of PAS-positive material.

The following histochemical reactions were also performed: reduced nicotinamide-adenine denucleotide tetrazolium reductase (Burstone, 1962) and adenosine triphosphatase at $\mathrm{pH} 9.4$ (Padykula and Herman, 1955) and $\mathrm{pH} 4.3$ (Brooke and Kaiser, 1970). The incidence of fibres rich and poor in mitochondrial enzymes was normal; $16 \%$ of fibres rich in mitochondrial enzymes were atrophic as compared with at most $10 \%$ in normal muscle (Kamieniecka, unpublished).

\section{ELECTROMYOGRAPHY}

The brachial biceps muscle showed signs of myopathy: the mean duration of motor unit potentials was diminished by $25 \%$ (Fig. 1); the mean amplitude was normal. The incidence of polyphasic potentials was $6 \%$ above the upper limit of normal. The pattern of recruitment during full effort was normal, the amplitude was diminished. Spontaneous activity was absent. In the medial vastus muscle the only abnormality was a diminished amplitude of the recruitment pattern during full effort.

Electrical and mechanical responses of $\mathbf{m}$. adductor pollicis evoked by supramaximal stimuli

The latency, the time course, and the amplitude of the pretetanic muscle action potentials, of the twitch tension and of the twitch tetanus ratio (0.1) were normal.

Twenty seconds after the onset of $20 / \mathrm{s}$ stimuli for $50 \mathrm{~s}$, the tetanic tension decreased to half, as did the amplitude of the action potential (Fig. 2). There was, however, no decrement in the electrical or mechanical responses in $3 / \mathrm{s}$ trains. The decrease in tetanic tension was greater than in a control subject of the same age and in two normal adults in whom the tension and the action potential amplitude decreased by at most $20 \%$.

CONTRACTURE IN THE ADDUCTOR POLLICIS MUSCLE After cessation of the tetanic stimuli the patient complained of marked pain. The residual tension was $480 \mathrm{~g}, 17 \%$ of the maximal tetanic tension. This contracture was not associated with muscle action potentials; it decreased exponentially (Fig. 3), resting tension being attained after about 10 minutes. In controls there was a small displacement of resting tension after the tetanus, probably due to slight displacement of the arm (Fig. 3, above, right dashed line).

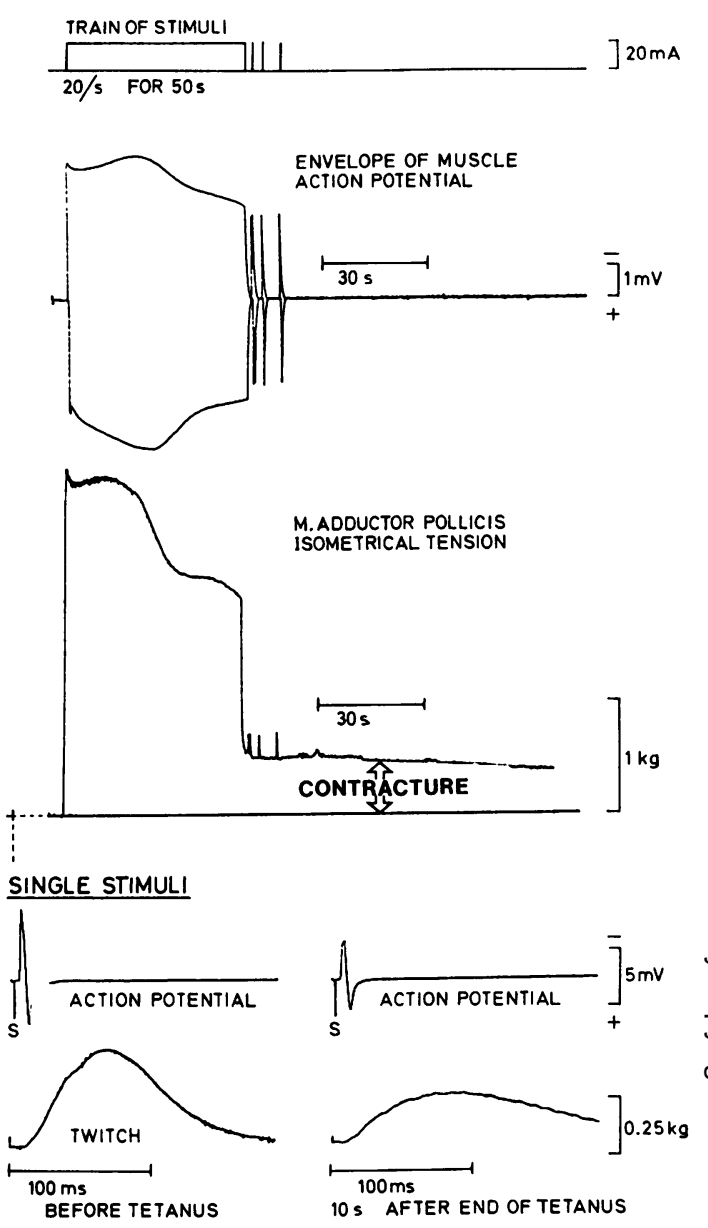

Fig. 2 Contracture after tetanus (without action potentials) in patient with McArdle's disease. Below: action potential associated with the twitch 10 seconds after the end of tetanus (right), the amplitude was half that of the pre-tetanic action potential and of the pre-tetanic twitch (left). Note the prolongation of the contraction time of the twitch after tetanus.

When the contracture was at its peak, single stimuli evoked twitches that had half the tension and half the action potential amplitude of pretetanic responses (Fig. 2). The contraction time of the twitch was prolonged by $59 \%$, whereas the duration of the muscle action potential was the same as before the tetanus. In controls the twitch tension was potentiated by $40-60 \%$ and the contraction time was prolonged at most $30 \%$. The action potential amplitude was the same as before the tetanus.

The twitch tension and the contraction time reached pre-tetanic levels three to four minutes 

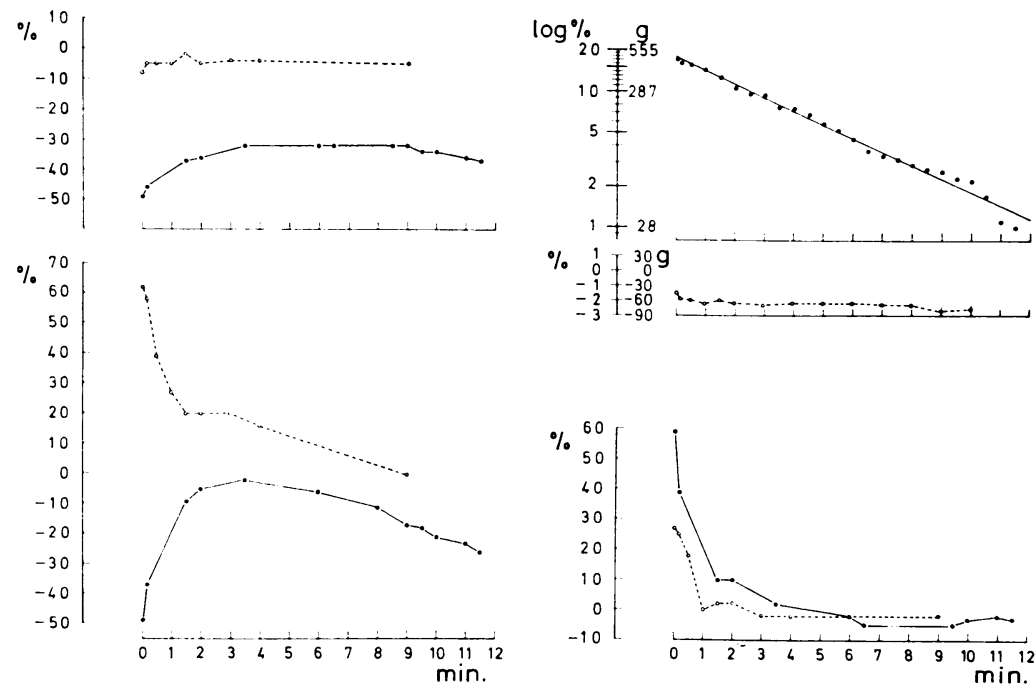

Fig. 3 Time course of mechanical tension and of electrical responses after a tetanus, 50 seconds in duration, in the adductor pollicis muscle of the patient with McArdle's disease (- $\longrightarrow$ and in normal subject of the same age (-...--). Above, left: decrease in the action potential amplitude associated with the twitch in the patient; the amplitude remained unchanged in the normal subject. Above, right: residual tension, 'cramp', logarithmic scale in the patient, linear scale in the normal subject (in grams and in per cent of the peak tetanic tension). Below, left: decrease in twitch tension in the patient (in per cent of the pre-tetanic twitch) and post-tetanic potentiation in the normal subject (at most $60 \%$ ). Below, right: the greater prolongation of contraction time in the patient than in the normal subject.

after cessation of the tetanus, when the contracture had decreased to half its peak value. The action potential did not return to the pre-tetanic amplitude within the time of observation.

Ninety seconds after cessation of the tetanus there was evidence of delayed $(50 \mathrm{~ms})$ repetitive firing associated with an additional peak in tension (Fig. 4).

Finally, $2 / \mathrm{s}$ stimuli were delivered for $90 \mathrm{~s}$, when the contracture had decreased to one-quarter of its peak value. There was no positive staircase phenomenon, the twitch tension and the action potential amplitude being unchanged. None the less the contraction time was shortened by $14 \%$, as it is during a positive staircase of normal muscle (Desmedt and Hainaut, 1968; Slomić et al., 1968).

CONTRACTURE IN THE FLEXORS OF THE ELBOW Maximal voluntary flexion produced a force of $9.3 \mathrm{~kg}$ at the wrist, the force decreasing to $6 \mathrm{~kg}$ after 74 seconds. Immediately after the patient was asked to relax the residual tension was $19 \%$ of the peak tetanic tension and decreased exponentially, more slowly than in the adductor pollicis muscle (decay constant 10.5 minutes as compared with 4.3 minutes in the adductor pollicis). The contracture was associated with marked pain, more pronounced than in the ad-

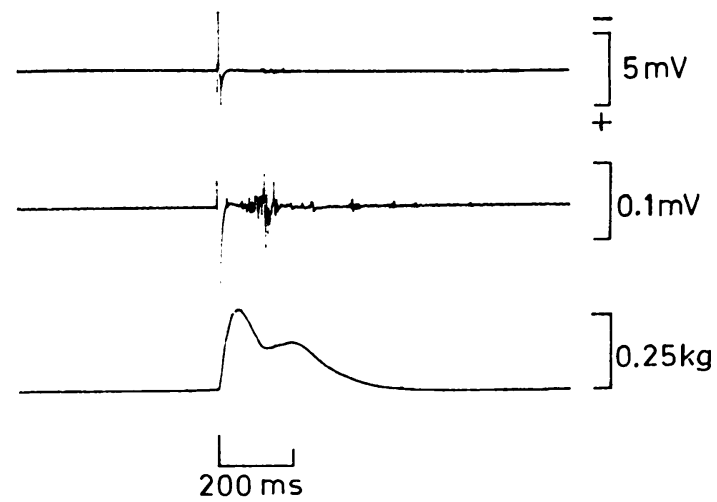

Fig. 4 Delayed firing evoked by a single stimulus observed first when the contracture had decreased to $80 \%$ of maximum. Upper and middle traces: muscle action potential on the middle trace recorded with 50 times higher amplification; lower trace: mechanical response; the delayed firing resulted in a second peak. 
ductor pollicis muscle and, due to displacement of the arm, the time when the pre-tetanic tension was reached could not be determined.

\section{Discussion}

The patient had the symptoms and signs of McArdle's disease: (i) vigorous exercise evoked painful cramps, not associated with electrical activity; (ii) on histochemistry, phosphorylase a and $b$ were absent and glycogen was increased; (iii) lactate and pyruvate did not increase in venous blood after exercise during ischaemia; (iv) eight times, firstly when the patient was 9 years old, vigorous exercise was associated with myoglobinuria.

Maximal stimuli to the nerve, given at a rate of $20 / \mathrm{s}$ for $50 \mathrm{~s}$ precipitated cramps not associated with muscle action potentials (Dyken et al., 1967). The tension developed during the cramp was onefifth of the maximal tetanic tension in the adductor pollicis muscle. That the contracture was of the same order in the brachial biceps after full voluntary effort suggested that it had attained maximum in both muscles.

At the peak of the contracture the twitch tension and the action potential concomitant with it were diminished to half. Then, if we assume that a contractured muscle fibre is unexcitable, half of the fibres participate in the contracture. The contracture was concomitant with a prolongation in contraction time, in non-contractured fibres to twice that in controls. This prolongation was not due to repetitive firing or to selective contracture of fast type II fibres which comprise $20 \%$ of the fibres in the adductor pollicis muscle (Johnson et $a l .$, 1973). Thus, both slow and fast fibres contribute to the prolongation of the contraction time as well as to the contracture.

Unlike most patients with McArdle's disease our patient showed electromyographic and mild histological signs of myopathy in the brachial biceps muscle. Moreover, serum creatine kinase was increased two and five months after episodes of myoglobinuria. Electromyographic evidence of myopathy has been described in McArdle's disease by Gruener et al. (1968) (one patient), by Delwaide et al. (1969) (two patients), and by de Castro et al. (1971) (one patient). That the staircase phenomenon was absent is further evidence of myopathy (Desmedt and Emeryk, 1968).

We wish to thank Dr J. Løchte for referring the patient with McArdle's disease. We also thank Dr P. Wang, Central Laboratory, Aarhus Community Hospital, for the biochemical analysis of the muscle biopsies. This work was supported by grants to FB from the Muscular Dystrophy Associations of America, Inc, and the Michaelsen Foundation, Copenhagen, and to NJB from the Danish Medical Research Council.

\section{References}

Brooke, M. H., and Kaiser, K. K. (1970). Muscle fiber types: how many and what kind? Archives of Neurology (Chicago), 23, 369-379.

Burstone, M. S. (1962). Enzyme Histochemistry. Academic Press: New York and London.

de Castro, A. R., Ricoy, J. R., Diaz-Flores, L., Gamuñez, A., and Lafuente, G. (1971). Un caso de miopatica de McArdle. Libro de resumenes de la XXIII Reunion annual de la Sociedad Española de Neurologia, Barcelona.

Delwaide, P. J., Simar, L., Penders, C. A., Lemaire, R., Lelievre, P., and Reznik, M. (1969). Contribution au diagnostic de la Maladie de MacArdle (avec description d'un nouveau cas). Acta Neurologica et Psychiatrica Belgica, 69, 272-284.

Desmedt, J. E., and Emeryk, B. (1968). Disorder of muscle contraction processes in sex-linked (Duchenne) muscular dystrophy, with correlative electromyographic study of myopathic involvement in small hand muscles. American Journal of Medi- $\bar{D}$ cine, 45, 853-872.

Desmedt, J. E., and Hainaut, K. (1968). Kinetics of myofilament activation in potentiated contraction: staircase phenomenon in human skeletal muscle Nature, 217, 529-532.

Dyken, M. L., Smith, D. M., and Peake, R. L. (1967). An electromyographic diagnostic screening test in McArdle's disease and a case report. Neurology (Minneapolis), 17, 45-50.

Ebbesen, F., Brandt, N. J., and Løchte, J. (1977). McArdle's syndrom. Ugeskrift for Laeger. In press.

Eränko, O., and Palkama, A. (1961). Improved localization of phosphorylase by the use of polyvinyl pyrrolodine and high substrate concentration. Journal of Histochemistry and Cytochemistry, 9, 585.

Godlewski, H. G. (1963). Are active and inactive phosphorylases histochemically distinguishable? Journal of Histochemistry and Cytochemistry, 11, 108-113.

Gruener, R., McArdle, B., Ryman, B. E., and Weller, R. O. (1968). Contracture of phosphorylase deficient muscle. Journal of Neurology, Neurosurgery, and Psychiatry, 31, 268-283.

Johnson, M. A., Polgar, J., Weightman, D., and Appleton, D. (1973). Data on the distribution of fibre types in thirty-six human muscles. An autopsy study. Journal of the Neurological Sciences, 18, 111-129.

Krebs, E. G., Love, D. S., Bratvold, G. E., Trayser, K. A., Meyer, W. L., and Fischer, E. H. (1964). Purification and properties of rabbit skeletal muscle phosphorylase b kinase. Biochemistry, 3, 1022-1033. 
Landon, J., Fawcett, J. K., and Wynn, V. (1962). Blood pyruvate concentration measured by a specific method in control subjects. Journal of Clinical Pathology, 15, 579-584.

Lundholm, L., Mohme-Lundholm, E., and Vamos, N. (1963). Lactic acid assay with L(+)lactic acid dehydrogenase from rabbit muscle. Acta Physiologica Scandinavica, 58, 243-249.

McArdle, B. (1951). Myopathy due to a defect in muscle glycogen breakdown. Clinical Science, 10, 13-35.

Padykula, H. A., and Herman, E. (1955). The specificity of the histochemical method of adenosine triphosphatase. Journal of Histochemistry and Cytochemistry, 3, 170-195.

Porte, D., Crawford, D., Jennings, D. B., Aber, C., and Mellroy, M. (1966). Cardiovascular and metabolic responses to exercise in McArdle's syndrome. New England Journal of Medicine, 275, 406-412.

Rowland, L. P., Fahn, S., and Schotland, D. L. (1963). McArdle's disease. Hereditary myopathy due to absence of muscle phosphorylase. Archives of Neurology (Chicago), 9, 325-342.

Slomić, A., Rosenfalck, A., and Buchthal, F. (1968). Electrical and mechanical responses of normal and myasthenic muscle, with particular reference to the staircase phenomenon. Brain Research, 10, 1-78.

Thomas, J. A., Schlender, K. K., and Larner, J. (1968). A rapid filter paper assay for UDP-glucose-glycogen glucosyltransferase, including an improved biosynthesis of UDP-14C-glucose. Analytical Biochemistry, 25, 486-499. 